University of Nebraska - Lincoln

DigitalCommons@University of Nebraska - Lincoln

1981

\title{
A Graph Model for Pattern-Sensitive Faults in Random Access Memories
}

Sharad C. Seth

University of Nebraska-Lincoln, seth@cse.unl.edu

K. Narayanaswamy

University of Nebraska-Lincoln

Follow this and additional works at: https://digitalcommons.unl.edu/csearticles

Part of the Computer Sciences Commons

Seth, Sharad C. and Narayanaswamy, K., "A Graph Model for Pattern-Sensitive Faults in Random Access Memories" (1981). CSE Journal Articles. 41.

https://digitalcommons.unl.edu/csearticles/41

This Article is brought to you for free and open access by the Computer Science and Engineering, Department of at DigitalCommons@University of Nebraska - Lincoln. It has been accepted for inclusion in CSE Journal Articles by an authorized administrator of DigitalCommons@University of Nebraska - Lincoln. 
doi: 10.1109/TC.1981.1675737

\section{Correspondence}

\section{A Graph Model for Pattern-Sensitive Faults in Random Access Memories}

\section{SHARAD C. SETH AND K. NARAYANASWAMY}

\begin{abstract}
This correspondence generalizes Hayes' recent ideas for generating an optimal transition write sequence which forms the "backbonẹ" of his algorithm for testing semiconductor RAM's for pattern-sensitive faults. The generalization, presented in graph theoretic terms, involves two sequential steps. The first step results in assigning of a "color" to each memory cell. In the second step, each color is defined as a distinct sequence of bits representing the sequence of states assumed by the correspondingly colored cell. The constraints imposed at each step lead to interesting and general problems in graph theory: the standard graph coloring problem in the first step, and a path projection problem from a binary $m$-cube to a subcube in the second step. Applications to arbitrary $k$-cell neighborhoods, and particularly to three-cell neighborhoods are shown.
\end{abstract}

Manuscript received December 19, 1979; revised April 20, 1981.

S. C. Seth is with the Department of Computer Science, University of Nebraska, Lincoln, NE 68588.

K. Narayanaswamy was with the Department of Computer Science, University of Nebraska, Lincoln, NE 68588. He is now with the Department of Computer Science, University of Southern California, Los Angeles, CA 90007.
Index Terms-Coloring algorithm, graph modeling, optimal transition write sequences, RAM testing, single pattern-sensitive faults.

\section{INTRODUCTION}

A pattern-sensitive fault is said to occur in a random access memory (RAM) whenever the reading or writing of a cell malfunctions in the context of a specific pattern of information stored in memory. This class of faults is particularly elusive because an apparently fixed operation involving a particular cell could sometimes work and malfunction at others, giving the illusion of an intermittent fault. The latency of such a fault may also be very long because the pattern causing it may occur only very infrequently. The most general model for pattern sensitivity is one in which no restrictions are placed on which cells could affect operations on which other cells. Unfortunately, test sequences derived on the basis of such a model grow exponentially with the memory size (Hayes [3]), and hence, are impractical. Apart from practical considerations, models that restrict pattern sensitivity to be a "local" phenomenon may also be justified on the basis of prior visual inspection (Mead and Conway [6, p. 46]).

In this correspondence, we follow one such model proposed by Hayes [4] and show how his ideas about test generation for single pattern-sensitive faults can be generalized. We concentrate on the problem of minimizing the length of transition write sequences, since 
these form the "innermost" loops in Hayes' algorithm for test generation. The graph formulation leads to an interesting connection of the problem with graph coloring and formulation of a new problem in graph projection.

Other models of pattern sensitivity have appeared in recent literature, e.g., [7]-[10].

\section{BACKGROUND}

This section is in the form of a brief review, summarizing concepts and notation relating to a fault model for pattern sensitivity as introduced in [3] and [4]. Only the material essential to this correspondence is included.

\section{A. The Fault Model}

An $n$-cell (one-bit wide) RAM $M_{n}=\left\{C_{0}, \cdots, C_{n-1}\right\}$ is treated as an incompletely specified sequential machine. $M_{n}$ has three input operations: read cell $C_{i}$, write 1 into $C_{i}$, and write 0 into $C_{i}$; these operations are denoted by $R_{i}, W_{i}$, and $\bar{W}_{i}$, respectively. $\tilde{W}_{i}$ is used to represent either $W_{i}$ or $\bar{W}_{i}$. When $M_{n}$ is fault-free, $R_{i}$ does not alter the state of $M_{n}$ (composed of the binary state of each cell in $M_{n}$ ), and yields an output value $z$ representing the contents or state $s_{i}$ of $C_{i} . W_{i}$ and $\bar{W}_{i}$ change $s_{i}$ only and leave $z$ unspecified. By letting $S$ stand for the set of memory states, one can also view $R_{i}$ and $\tilde{W}_{i}$ as affecting mappings as follows:

$$
\begin{gathered}
R_{i}: S \rightarrow S \times\{0,1\} \\
\tilde{W}_{i}: S \rightarrow S \times\{-\}
\end{gathered}
$$

where "-" denotes an unspecified entry. A pattern-sensitive fault (PSF) allows $R_{i}$ and $\tilde{W}_{i}$ to become arbitrary functions from $S$ to $S \times$ $\{0,1,-\}$. In a single PSF (SPSF) only the operations addressed to one cell can be faulty. Test generation for SPSF's is complicated because reading and writing operations on a cell must be verified independently for each memory state. The state diagram of the incompletely specified machine $M_{n}$ will be found convenient for developing test-generation algorithms. The state diagram is a directed graph (a binary $n$-cube) with $2^{n}$ nodes and $3 n \cdot 2^{n}$ edges; each node is labeled with a distinct state of $M_{n}$ and each edge is labeled with the combination of a memory operation $\left(R_{i}, W_{i}\right.$, or $\left.\bar{W}_{i}\right)$ and the resultant output $(0,1$, or -$)$. Failures associated with an SPSF may be ascribed to the corresponding edge(s) on this graph. Associated with any test sequence of read and write operations is a path on the graph (that is, a sequence of directed edges for which the terminal node of one edge is the initial node of the next edge in the sequence). To trace the path, one simply starts at the node labeled with the initial state assumed for the test and follows sequentially the edges marked with the input operations defining the test sequence. The path could alternatively be described by a state sequence, that is, by the sequence of nodes traversed. The state sequence has the property that adjacent entries in the sequence differ at most in one-bit position.

\section{B. Near-Minimal Tests for SPSF's-Unrestricted Neighborhoods}

It should be clear that any algorithm aimed at reducing the test length must include an efficient way of traversing all the edges in the state diagram. Fortunately, a well-known result in graph theory due to Euler (cf. [5, p. 176]) guarantees the existence of a path which includes each edge once and only once for the memory state diagrams. Such a path is called Eulerian. An algorithmic way of obtaining an Eulerian path need not consider, to begin with, any of the nontransition edges - those that do not result in a change of state-since these are added easily to a path which includes all and only the transition edges. A simple but clever algorithm to obtain such an optimal transition write sequence appears as Procedure 1 in $[4, \mathrm{p}$. 250], so it will not be repeated here. In further discussion, it will be assumed that an optimal transition-write sequence $\mathcal{N}_{n}$ and the corresponding state sequence $\mathcal{S}_{n}$ has already been derived for $M_{n}$. From $\mathcal{f}_{n}$, other optimal state transition sequences can be derived in a way that would be useful to us later on. The state vector $\mathcal{S}_{n}$ is viewed as $n$ parallel one-bit sequences denoted conveniently by members of the integer set $Z_{n}=\{0,1, \cdots, n-1\}$. Reassigning cells of $M_{n}$ with a different pattern of integers corresponds to a different way of traversing an Eulerian path on the state diagram.

Procedure 1 (Adopted from Procedure 3 in [4]): To construct a test sequence $\mathcal{C}_{n}$ for SPSF's in $M_{n}$.

a). Follow each $\tilde{W}_{i}$ in $\mathcal{N}_{n}$ by another copy of $\tilde{W}_{i}$, thus introducing all nontransition writes.

b) Follow each $\tilde{W}_{i}$ (which is applied to cell $c_{i}$ ) by a sequence $\mathcal{R}$ of $n$ reads that read every cell in the memory. If only write-SPSF's had been assumed the sequence up to this point would constitute an optimal-length test.

c) Follow one application of $\mathscr{R}$ to $M_{n}$ when the latter is in state $S_{j}$ by a second copy of $\mathscr{R}$. Repeat for all $S_{j}$. This ensures testing of read-SPSF's but in a nonoptimal way.

The length $\left|\mathcal{C}_{n}\right|$ of $\mathcal{C}_{n}$ is given by

$$
\left|\mathcal{C}_{n}\right|=(3 n+2) 2^{n} \cdot n \text {. }
$$

\section{Neighborhood Restrictions}

The presence of the exponential term in (1) for test length appears to make the whole approach impractical for testing real memories. A little reflection shows that the exponential term arises due to the stipulation that operations on a cell may fail because of the bit stored in any other cell in the memory. In practice, one would expect pattern sensitivity to be much more localized. That is, only a few cells in close physical (or "logical") proximity of a cell $C_{i}$ can affect operations addressed to $C_{i}$. These cells are said to form the neighborhood $N\left(C_{i}\right)$ of $C_{i}$ (it is customary to include $C_{i}$ in its own neighborhood). Two further restrictions, somewhat less easily justified on practical grounds, will be imposed on neighborhoods. The first is that all cell neighborhoods are the same size $k$ (where, as assumed above, $k \ll$ $n$ ), and the second, that the cell neighborhoods are geometrically regular in some $d$-dimensional Euclidian space (usually a plane). The latter restriction may also be stated by saying that given one neighborhood, any other neighborhood can be obtained by its linear translation (without reflection or rotation). The question of the "boundary" neighbprhoods (those for which linear translation falls partially outside the physical dimensions of the memory) is left unaddressed in this model. Possible solutions include end-to-end folding of each dimension of the Euclidean space, or adding of dummy cells at the boundaries. Contiguity of cells within a neighborhood may be assumed from practical considerations, but is not really essential.

A compact notation for representing cell neighborhoods follows from the restriction of geometrical regularity. All $k$-cell neighborhoods of a type in a $d$-dimensional organization of $M_{n}$ can be derived from a template $T_{k}$ of $k d$-dimensional (column) vectors $V_{i}$ 's

$$
T_{k}=\left(V_{0}=(0,0, \cdots, 0), V_{1}, \cdots, V_{k-1}\right) \text {. }
$$

To obtain the neighborhood of a cell $C_{I}$, where $I$ is the $d$-dimensional index of the cell, one simply adds the vectors $V_{0}, V_{1}, \cdots$, and $V_{k-1}$ in turn to the vector $I$ to derive cell indices of the neighborhood cells. For example, the template

$$
\dot{T}_{5}=((0,0),(0,1),(0,-1),(1,0),(-1,0))
$$

describes the standard five-cell neighborhood in a two-dimensional memory, consisting of the north, south, east, and west neighbors of a cell.

Near-optimal test sequences for SPSF's under the neighborhood restrictions can still be derived by a procedure very similar to procedure 1 , but with the following modifications.

1) By far the most crucial change occurs in the initial sequence $\mathcal{N}_{n}$, which must now ensure traversal of transition writes within each of the $n k$-cell neighborhoods. A nonoptimal way to do this would be to treat each neighborhood independently and derive a locally optimal transition write sequence for it using Hayes' Procedure 1. This provides the upper bound $n\left(k \cdot 2^{k}\right)$ for the initial sequence $\mathcal{N}_{n}$ in Procedure 1. A lower bound of $n \cdot 2^{k}$ (achieved in [4] only for a special class of neighborhoods-see next section) is derivable by considering 
the maximum possible overlap in traversing neighborhoods: since a cell can belong to no more than $k$ neighborhoods, the upper bound can at best be improved by a factor of $k$.

2) The first sentence of step b) of the procedure must be changed to read: "Follow each $\tilde{W}_{i}$ (which is applied to cell $C_{i}$ ) by a sequence $\mathscr{R}_{i}$ of $k$ reads that read every cell in the neighborhood $N\left(C_{i}\right)$."

3) The first two sentences of step c) must be replaced by: "Follow one application of $\mathscr{R}_{i}$ to $N\left(C_{i}\right)$, when the latter is in state $S_{j}$ by a second copy of $\mathscr{R}_{i}$. Repeat for all $C_{i}$ and $S_{j}$."

It is easily shown that with these modifications, the length $\left|\mathscr{C}_{n}\right|$ is bounded as follows:

$$
(3 k+2) 2^{k} \cdot n \leq\left|e_{n}\right| \leq(3 k+2) k \cdot 2^{k} \cdot n .
$$

\section{Generation of Optimal Transition Write Sequences}

The lower bound of $n \cdot 2^{k}$ for the length of $\mathcal{N}_{n}$ is achievable for the special class of tiling neighborhoods on a plane. The template for a tiling neighborhood is defined to satisfy two restrictions:

1) it should be a figure formed by joining squares along their edges, i.e., a polyomino [2],

2) it should be capable of tiling the plane by simple translation.

Lemma 1 (Lemma 1 in [4]): Let $N$ be a $k$-cell tiling neighborhood of $M_{n}$. Each cell of $M_{n}$ can be marked with an integer from the set $Z_{k}=\{0,1, \cdots, k-1\}$ in such a way that every neighborhood of type $N$ in $M_{n}$ is marked with all members of $Z_{k}$.

An optimal transition write sequence is derived from a marking of $M_{n}$ in two steps. In the first step, each integer is replaced by its associated bit-sequence of length $k \cdot 2^{k}$ derived from $\oint_{k}$ as discussed in Section II-B. The composed sequence for $M_{n}$, however, involves $n / k$ transition writes between adjacent rows because all cells marked with the same integer would change their states simultaneously. This is remedied in the second step by replacement of simultaneous writes by a sequence of single writes. The resultant sequence has the length $(n / k) k \cdot 2^{k}=n \cdot 2^{k}$.

\section{TRansition Write SEQuences for ARbitraRY NEIGHBORHOODS}

The essential point to note about the preceding discussion is that the problem of finding an optimal transition write sequence has been reduced to that of numbering memory cells from an integer set, such that no two cells belonging to the same neighborhood are numbered identically. Lemma 1 shows that for tiling neighborhoods of $k$ cells, this is possible using only $k$ distinct numbers. We generalize the procedure to nontiling neighborhoods by using $k$ or more distinct numbers. As a motivating example, consider three-cell neighborhoods for a linear array of cells defined by the template $T_{3}=(0,1,3)$. Simple trial and error will convince the reader that no numbering from the set $Z_{3}$ is possible; ${ }^{1}$ however, a very simple numbering exists from the set $Z_{4}$ (Fig. 1) which ensures that any pair of cells sharing a neighborhood are numbered differently. All four combinations of three integers occur for different neighborhoods in this example. The next step in the extension of the procedure is to associate a distinct but equilength one-bit column vector $B_{i}$ with each integer $i$ in the set $Z_{4}$, so that the matrix $\mathscr{S}=\left(B_{0}, B_{1}, B_{2}, B_{3}\right)$ satisfies the following two constraints:

1) adjacent rows of $\mathscr{S}$ involve a single transition write, and

2) $\mathcal{S}$ traverses (at least once) each transition-write edge in the state diagram for every three-cell memory $m_{3}$ obtained by considering three cells numbered differently from each other.

One such possibility for bit-vectors is shown in Table II. Thus, the procedure outlined at the end of Section II can be extended to generate a transition write sequence for this nontiling neighborhood. The optimality of this sequence is proved at the end of Section III-B.

\footnotetext{
${ }^{1}$ We state, without proof, the following lemma hinting at the "sparseness" of tiling neighborhoods. It is arguable, however, that a much larger fraction of the neighborhoods of "practical" interest may be tiling.

Lemma 2: Three-cell neighborhoods defined by templates of the form $(0$, $1, p)$ are tiling if and only if $p$ (modulo 3$)=2$.
}

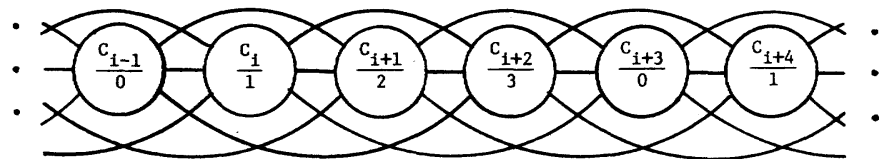

Fig. 1. The neighbor-of graph for the template $T_{3}=(0,1,3)$ and a repetitive numbering scheme using $Z_{3}=\{0,1,2,3\}$.

\section{A. The Graph Model}

A formal statement of the ideas embodied in the above example is perhaps best phrased in terms of graph theory. In the discussion below, we limit ourselves to linear neighborhoods because there exist well-known mappings (e.g., the row-major or the column-major) that can be used to obtain a linear template from a higher dimensional template once the array dimensions are known.

Cell $C_{i}$ is said to be a neighbor of cell $C_{j}$ (denoted as $C_{i} \mathcal{N} C_{j}$ ) if $C_{i}$ and $C_{j}$ share a common neighborhood. The binary relation $\mathcal{N}$ is reflexive and symmetric but not transitive because of the possibility that neighborhoods overlap only partially. Associated with $\mathcal{N}$ is the graph of the relation $G(\mathcal{N})$, which has $n$ nodes (one for each memory cell) and undirected edges between cells which are neighbors of each other.

The graph model provides an elegant generalization of the two steps described in the above example for deriving a transition write sequence.

Step 1: This is equivalent to numbering of cells in the example. In terms of $G(\mathcal{N})$ the restriction on numbering becomes the standard graph coloring problem. Color the nodes of the graph in such a way that no two nodes connected by an edge (i.e., adjacent nodes) are colored identically. For $k$-cell neighborhoods, at least $k$ colors will be necessary, since all the nodes for cells in a neighborhood will be adjacent on the graph. Hayes' result (Lemma 1) may be restated by saying that $k$ colors are sufficient for tiling neighborhoods. Nontiling neighborhoods may require $m>k$ colors. In order not to proliferate notation we will continue to use the integer set $Z_{m}$ to represent the required $m$.colors.

Step 2: This step is equivalent to association of one-bit (column) vectors with the integers in the exampie. The rows of the matrix $\delta=$ $\left(B_{0}, B_{1}, \cdots, B_{m-1}\right)$ should be in the Gray-code sequence. Further, if $\left\{i_{0}, i_{1}, \cdots i_{k-1}\right\}$ corresponds to the set of (distinct) integers of an arbitrary neighborhood $N$, then the rows of the matrix $\mathcal{f}^{\prime}=\left(B_{i_{0}}, B_{i_{1}}\right.$, $\cdots, B_{i_{k-1}}$ ) must traverse the neighborhood $N$ in such a way that every transition write is included at least once. $\mathcal{S}^{\prime}$ will be called a $k$-dimensional projection of the $m$-dimensional sequence $\mathcal{S}$. We note that while the above restriction may not be necessary for every $k$-dimensional projection of $\mathcal{S}$ (because a particular combination of $k$ integers may not be assigned to any neighborhood by a coloring scheme), requiring it will certainly be sufficient and perhaps, mathematically more tractable.

What about the optimality of a transition write sequence derived using the above two steps? This is essentially determined by the number of rows in the matrix $\mathcal{S}$. Obviously, $\mathcal{S}$ need not be longer than if it were derived for unrestricted neighborhoods in $M_{m}$. That is

$$
\left|\delta^{\prime}\right| \leq\left|\delta_{m}\right|=m \cdot 2^{m}+1 .
$$

Further, $\rho$ could not be shorter than $m \cdot 2^{k}+1$; otherwise the lower bound of $n \cdot 2^{k}$ would be violated for a transition write sequence for the whole memory $M_{n}$. Thus, the endeavor in the second step should be to arrive at a matrix $\delta$ with the number of rows equal to this lower bound. Intuitively, it appears that optimality is easier to achieve (or approach) in the second step if $m$ is as small as possible, that is, one cannot afford to be profligate in the use of colors in the first step.

\section{B. Arbitrary $k$-Cell Neighborhoods}

In this section, we provide partial solutions to the problem of deriving a near-optimal transition write sequence for arbitrary $k$-cell neighborhoods using the graph model described above.

Minimum Number of Colors: Let $m$ be the minimum number of colors required for $k$-cell neighborhoods defined by the (linear) template $T_{k}=\left(V_{0}=0, V_{1}, \cdots, V_{k-1}\right)$ where, without loss of gen- 
TABLE I

“WORST CASE" NEIGHBORHOODS

\begin{tabular}{|c|c|c|}
\hline Neighborhood size (k) & Template $\left(T_{k}\right)$ & Minimum number of colors (m) \\
\hline 3 & $(0,1,3)$ & 4 \\
\hline 4 & $(0,2,5,6)$ & 7 \\
\hline 5 & $(0,3,4,8,10)$ & 9 \\
\hline 6 & $(0,4,6,7,10,15)$ & 12 \\
\hline
\end{tabular}
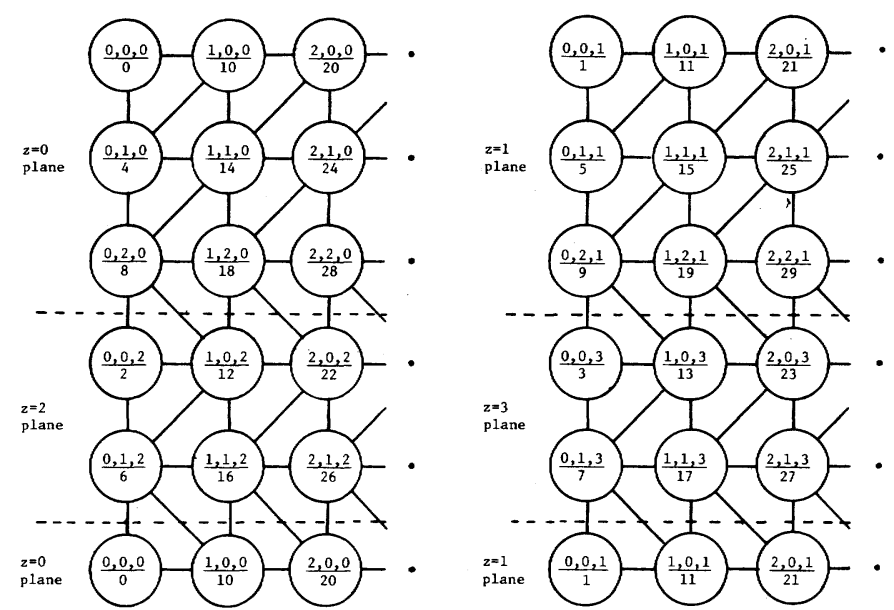

Fig. 2. Two components of the neighbor-of graph for the template $T_{3}=$ $(0,4,10)$; linear cell index at the bottom and three-dimensional index on the top.

erality, we assume that $V_{i}>V_{j}$ if $i>j$. As noted above, $m$ cannot be smaller than $k$. However, this lower bound on $m$ is often quite weak and can be improved by examining the template more closely. One possible improvement is indicated in the following theorem.

Theorem 1: Let

$$
\Delta_{T_{k}}=\left\{V_{i}-V_{j}: i>j\right\}
$$

be the set of pairwise differences of numbers in $T_{k}$. Consider all arithmetic series formed by choosing integers from the set $\Delta_{T_{k}}$, and let $p$ be the length of the longest such series. Then the minimum number of required colors $m \geq p$.

Proof: The proof of the theorem follows by showing that every pair of cells in the set $\left\{C_{a}, C_{a+r}, \cdots, C_{a+(p-1) r}\right\}$ are mutual neighbors, where $(a, a+r, \cdots, a+(p-1) r)$ is (one of) the longest series.

Table I shows some of the worst case neighborhoods derived by using the theorem for small values of $k$. It is conjectured that there are no $k$-cell neighborhoods requiring more than the maximum number of colors indicated by this theorem. Further, we conjecture that this worst case bound is linear in $k$ and is given by the floor function $\lfloor(5 k-6) / 2\rfloor$.

Upper bounds on $m$ can be found constructively by considering specific coloring schemes. An attractive scheme for algorithmic implementation is the cyclically repeating pattern $(0,1, \cdots, m-1)$ such as used in Fig. 1. In this scheme, cell $C_{i}$ is colored with the integer $i$ (modulo $m$ ). For the scheme to work it can be shown by elementary number theory that $m$ must be such that

$$
V_{i}(\operatorname{modulo} m) \neq V_{j}(\operatorname{modulo} m), \quad i \neq j .
$$

The smallest such $m$ provides an upper bound on the number of colors required. For example, it can be easily verified that for the template $T_{6}=(0,4,6,7,10,15)$ the smallest such $m$ is 12 so the memory cells can be colored with the cyclically repeating pattern $(0,1, \cdots, 11)$.
From Table I we also know that no coloring scheme with less than 12 colors can exist, so the cyclic pattern is an optimal way to color the cells.

Color Definition in Terms of One-Bit Column Vectors: The general problem of defining $m(>k)$ colors optimally in terms of one-bit vectors remains unsolved. However, a solution has been found when $m$ exceeds the neighborhood size $k$ by just one and is even. We need the following definition to describe the solution.

Consider the $m$-cube as an undirected graph and let $e_{1}=\left\{X_{1}, Y_{1}\right\}$ and $e_{2}=\left\{X_{2}, Y_{2}\right\}$ be its two distinct edges, such that the nodes $X_{1}$ and $X_{2}$ are adjacent. Then we call edges $e_{1}$ and $e_{2}$ conjugates of each other whenever the nodes $Y_{1}$ and $Y_{2}$ are also adjacent.

The essential step in the solution assigns a unique direction to each edge of the $m$-cube in such a way that: 1) for each vertex there are exactly $m / 2$ edges directed to and away from it, and 2) conjugate edges are assigned opposite directions. Then the Eulerian theorem $[5$, p. 176] guarantees existence of an Eulerian path on the resultant directed graph. Further, because conjugate edges are assigned opposite edges, it is easily seen that a transition write sequence is included for every $(m-1)$-dimensional projection of the state sequence associated with the Eulerian path.

Procedure (To Assign Directions to the Edges of an m-Cube):

Step 1 (Assign Directions to all the Edges Incident on a Fixed Node $)$ : Let $X=\left(x_{0}, \cdots, x_{m-1}\right)$ be a fixed node and let node $Y$ be adjacent to $X$. Then there is exactly one $i \in\{0, \cdots, m-1\}$ for which $y_{i}=\bar{x}_{i}$. If $i$ is even assign the edge $\{X, Y\}$ the direction $X \rightarrow Y$; else assign it the opposite direction.

Step 2 (Assign Directions to the Remaining Edges): Recursively apply the following rule until all the edges are assigned directions.

Assume an edge $e=\{X, Y\}$ has already been assigned the direction $X \rightarrow Y$, but not an edge $e^{\prime}$ which is a conjugate of $e$. Let $e^{\prime}=\left\{X^{\prime}\right.$, $\left.Y^{\prime}\right\}$, where without loss of generality we can assume that $X$ and $X^{\prime}$ are adjacent. Assign the direction $Y^{\prime} \rightarrow X^{\prime}$ to $e^{\prime}$.

It can be proved that the procedure assigns directions to edges in such a way that the two restrictions stated above are satisfied. The opti- 
TABLE II

OPTIMUM COLOR DEFINITION WHEN $m=4$ AND $k=3$

\begin{tabular}{|c|c|c|c|c|c|c|c|c|c|c|c|c|c|c|c|c|c|c|c|c|c|c|c|c|c|c|c|c|c|c|c|c|c|c|}
\hline & & & & & & & & & & & & & & tat & $\mathrm{s}$ & equ & enc & & & & & & & & & & & & & & & & & \\
\hline & & $\mathrm{s}_{0}$ & & . & $\cdot$ & · & & & & & & & . & . & - & - & & & & & & & & & & & & & & . & • & . & . & $\mathrm{s}_{32}$ \\
\hline & $\mathrm{B}_{0}$ & 0 & 1 & 1 & 1 & 1 & 1 & 1 & 1 & 1 & 0 & 0 & 1 & 1 & 1 & 1 & 0 & 0 & 0 & 0 & 0 & 0 & 1 & 1 & 0 & 0 & 0 & 0 & 1 & 1 & 0 & 0 & 0 & 0 \\
\hline \multirow[t]{3}{*}{$\begin{array}{c}\text { Bit } \\
\text { Vector: }\end{array}$} & $\mathrm{B}_{1}$ & 0 & 0 & 0 & 0 & 0 & 0 & 1 & 1 & 1 & 1 & 0 & 0 & 1 & 1 & 0 & 0 & 0 & 0 & 0 & 0 & 1 & 1 & 1 & 1 & 1 & 1 & 1 & 1 & 0 & 0 & 1 & 1 & 0 \\
\hline & $\mathrm{B}_{2}$ & 0 & 0 & 1 & 1 & 0 & 0 & 0 & 0 & 1 & 1 & 1 & 1 & 1 & 1 & 1 & 1 & 0 & 0 & 1 & 1 & 1 & 1 & 0 & 0 & 1 & 1 & 0 & 0 & 0 & 0 & 0 & 0 & 0 \\
\hline & $\mathrm{B}_{2}$ & 0 & 0 & 0 & 1 & 1 & 0 & 0 & 1 & 1 & 1 & 1 & 1 & 1 & 0 & 0 & 0 & 0 & 1 & 1 & 0 & 0 & 0 & 0 & 0 & 0 & 1 & 1 & 1 & 1 & 1 & 1 & 0 & 0 \\
\hline
\end{tabular}

mality of the transition write sequence associated with the Eulerian path follows from its length, $m \cdot 2^{m-1}$, which equals the lower bound $m \cdot 2^{k}$ when $k=m-1$.

\section{Arbitrary Three-Cell Neighborhoods}

The graph model has been most successfully applied to arbitrary three-cell neighborhoods. An optimal transition write sequence can always be derived in this case even for nontiling neighborhoods.

Minimum Number of Colors: Let $T_{3}=(0, p, q)$ represent an arbitrary three-cell template, where $0<p<q$. We define a mapping from the linear index $i$ of an arbitrary cell $C_{i}$ to a three-dimensional index $(x, y, z)$ with the aim of bringing all the neighboring cells close together in the three-dimensional space. Let

$$
\begin{aligned}
& x=\lfloor i / q\rfloor \\
& y=\lfloor i(\operatorname{modulo} q) / p\rfloor \\
& z=\{i(\operatorname{modulo} q)\}(\operatorname{modulo} p) .
\end{aligned}
$$

The mapping is invertible, since $i=q x+p y+z$. Fig. 2 shows the neighbor-of graph for the example $T_{3}=(0,4,10)$. With the above mapping of cell indices, the graph is seen to consist of two identical but disjoint components. Within each component the pattern of edge connections are regular, particularly if the graph is assumed to be drawn on a cylinder so that the bottom row is adjacent to the top row. The example can be generalized to any three-cell template-we state below the general results.

1) Let $g=\operatorname{gcd}(p, q)$. The graph then contains exactly $g$ identical but disjoint components.

2) Cells $C_{i_{1}}$ and $C_{i_{2}}$ belong to the same component iff $g$ divides $\left|z_{1}-z_{2}\right|$.

3) Each graph component has $p / g$ distinct $z$-planes (i.e., cells whose $z$-indices are identical) and $q / g$ distinct rows.

4) Within each $z$-plane, the edges connect the cells next to each other in the $x$ - and $y$-directions. In addition, cells directly across $+45^{\circ}$-diagonal from each other are also connected. This pattern is broken for connections between the bottom row of one $z$-plane and the top row of the next in only that the diagonal connections are between cells across the $-45^{\circ}$ diagonal.

The proofs of these assertions are omitted for brevity. Evidently then, the neighbor-of graph can be drawn on the surface of a cylinder without crossing of edges. Such graphs have been shown recently to be four-colorable [1]. ${ }^{2}$

Color Definition: From the above discussion $m$, the number of colors, is restricted to be either 3 or 4 . When $m=3$, Procedure 1 in [4] can be applied directly to define colors in terms of one-bit vectors. On the other hand, when $m=4$ and $k=3$, the restriction stated in

\footnotetext{
${ }^{2}$ The authors have also obtained a constructive proof for the sufficiency of four colors; it shows that one of two specific coloring schemes would always suffice. The coloring schemes and the proof are being omitted here because of their length.
}

Section III-B is satisfied so the procedure given in that section can be used to assign directions to the edges of the four-cube. Once this is done, an Eulerian path can be algorithmically derived [5, p. 175]. The color definition resulting from one such Eulerian path is shown in Table II. It is worth pointing out that the color definition step is essentially independent of any specific three-cell template and hence the color definition of Table II is applicable to any three-cell template requiring four colors.

\section{REFERENCES}

[1] K. Apple and W. Haken, "Every planar graph is four-colorable," Bull. Amer. Math. Soc., vol. 82, pp. 711-712, 1976; also in Sci. Amer., vol. 237, pp. 108-121, Oct. 1977.

[2] S. W. Golomb, Polyominoes. New York: Scribner, 1965.

[3] J. P. Hayes, "Detection of pattern-sensitive faults in random access memories," IEEE Trans. Comput., vol. C-24, pp. 150-157, Feb. 1975.

[4] — "Testing memories for single pattern-sensitive faults," IEEE Trans. Comput., vol. C-29, pp. 249-254, Mar. 1980.

[5] C. L. Liu, Introduction to Combinatorial Mathematics. New York: McGraw-Hill, 1968.

[6] C. Mead and L. Conway, Introduction to VLSI Systems. Reading, MA: Addison-Wesley, 1980.

[7] R. Nair, S. M. Thatte, and J. A. Abraham, "Efficient algorithms for testing semiconductor random access memories," IEEE Trans. Comput., vol. C- 27, pp. 572-576, June 1978.

[8] V.P. Srini, “API tests for RAM chips," Computer, vol. 10, pp. 32-35, July 1977.

[9] D. S. Suk and S. M. Reddy, "Test procedures for a class of pattern sensitive faults in semiconductor random access memories," IEEE Trans. Comput., vol. C-29, pp. 419-429, June, 1980.

[10] S. M. Thatte and J. A. Abraham, "Testing of semiconductor random access memories," in Proc. 7th FTCS, Los Angeles, CA, June 1977, pp. $81-87$. 